\title{
What counts as evidence for a logical theory?*
}

\author{
Ole Thomassen Hjortland \\ ole.hjortland@uib.no
}

\begin{abstract}
Anti-exceptionalism about logic is the Quinean view that logical theories have no special epistemological status, in particular, they are not self-evident or justified a priori. Instead, logical theories are continuous with scientific theories, and knowledge about logic is as hard-earned as knowledge of physics, economics, and chemistry. Once we reject apriorism about logic, however, we need an alternative account of how logical theories are justified and revised. A number of authors have recently argued that logical theories are justified by abductive argument (e.g. Gillian Russell, Graham Priest, Timothy Williamson). This paper explores one crucial question about the abductive strategy: what counts as evidence for a logical theory? I develop three accounts of evidential confirmation that an anti-exceptionalist can accept: (1) intuitions about validity, (2) the Quine-Williamson account, and (3) indispensability arguments. I argue, against the received view, that none of the evidential sources support classical logic.
\end{abstract}

\section{Introduction}

When we reject the apriority of logic, we are left with a less glamorous alternative. Basic logical knowledge is as hard-earned as the knowledge of physics, chemistry, or economics. The fortunes of logic are no less tied to $a$

\footnotetext{
*Acknowledgements: This research is funded by the Norwegian Research Council (FRIPRO grant 251218). Earlier drafts of this paper has been presented at the Munich Center for Mathematical Philosophy (MCMP) at LMU Munich, the 5th Young Researchers Days in Logic, Philosophy and History of Science, Académie Royale, Brussels, and the Logic As Science workshop at the University of Bergen. I am very grateful to all the participants for lively discussion. Special thanks to the many people who have discussed the material with me: Pål Antonsen, Aaron Cotnoir, Antony Eagle, Matti Eklund, Bruno Jacinto, Ragnhild Jordahl, Bruno Leclercq, Øystein Linnebo, Carlo Nicolai, Lavinia Piccolo, Graham Priest, David Ripley, Luis Rosa, Gillian Russell, Sindre Søderstrøm, Pilar Terrés, Peter Verdée, and Jack Woods.
}

Australasian Journal of Logic (16:7) 2019, Article no. 4 
posteriori evidence than other sciences. In Quine's phrase, logic is as revisable as the natural sciences:

$[\mathrm{N}]$ o statement is immune to revision. Revision even of the logical law of excluded middle has been proposed as a means of simplifying quantum mechanics, and what difference is there between such a shift and the shift whereby Kepler superseded Ptolemy, or Einstein Newton, or Darwin Aristotle. (Quine 1951, 40)

This is anti-exceptionalism about logic, a view more recently defended by Maddy (2002, 2014), Priest (2006a; 2014; 2016), Bueno \& Colyvan (2004), Russell (2014; 2015), and Williamson (2013a; 2013b; 2017). ${ }^{1}$ At the heart of the position are three familiar Quinean claims: gradualism, that logical theories are continuous with non-logical scientific theories; revisionism, that logical theories are revisable by pretty much by the same standards as other theories; and nonapriorism, that logical theories ultimately answer to $a$ posteriori evidence. $^{2}$

The anti-execeptionalist picture raises a number of questions. In what follows I aim to answer three:

(i) What is a logical theory a theory of?

(ii) What counts as evidential (dis)confirmation of a logical theory?

(iii) Does anti-exceptionalism provide support for classical logic?

The backdrop will be some recent attempts at developing an anti-exceptionalist account of theory-choice for logic. Roughly, the claim is that theory-choice in logic is justified by abductive argument. Let us call this position abductivism. For instance, one logical theory - say, a paracomplete theory - rejects the law of excluded middle, while the classical logical theory accepts it. According to abductivism, whether we opt for the paracomplete or the classical theory, will be a matter of inference to the best explanation. What counts as the best explanation will in turn depend on a number of theoretical virtues, for

\footnotetext{
${ }^{1}$ Some of the authors prefer to label themselves naturalists about logic. On my account, naturalism is a special case of anti-exceptionalism (cf. Hjortland 2017). According to naturalism, however, logical theories are not just continuous with, but rather subjugated by the sciences.

${ }^{2}$ Not all evidence for a logical theory need be a posteriori. Anti-exceptionalism only rules out the evidence being exclusively a priori. See Hjortland (2019) for a further discussion.
}

Australasian Journal of Logic (16:7) 2019, Article no. 4 
instance, fit with the evidence, simplicity, strength, conservativeness, and unification, just to mention a few.

The three questions take on a particular form in the context of abductivism. First, if logical theories are selected by inference to the best explanation, what is being explained by the theories? Second, fit with the evidence is a major criterion for abduction. But what counts as permissible evidence for the anti-exceptionalist? Third, Quine claims that anti-exceptionalism supports classical logic, a theory that according to him 'enjoys an extraordinary combination of depth and simplicity, beauty and utility' (Quine 1969, 11213). ${ }^{3}$ Correspondingly, Williamson argues that once we adopt abductivism as a method for theory-choice, classical logic is singled out as the best theory. Will Quine and Williamson's classicism be vindicated by abductivism? I will argue that the answer is 'no'.

In Section 2, I provide an account of logical theories that fits the antiexceptionalist agenda, and that is simultaneously broad enough to encompass major disagreements about logic. In Section 3, I discuss three potential sources of evidential confirmation for a logical theory: intuitions about validity, the Quine-Williamson account, and indispensability arguments. I conclude that neither of them offer convincing grounds for classical logic. Finally, in Section 7, I discuss to what extent certain non-evidential critera (strength, simplicity, conservativeness) favour classical logic, before I turn to a brief conclusion in Section 8.

\section{Logical theories}

What is a logical theory? To a first approximation, a logical theory is a theory of logical properties. The main logical property, most would agree, is validity. One chief function of a logical theory, then, is to tell us which arguments are valid. After all, philosophers of logic routinely disagree about whether or not arguments of a certain type are valid. Relevantists claim that disjunctive syllogism is invalid, paracompletists reject the law of excluded middle, while classicists accept both as valid. An account of logical theories should therefore reflect that there are rival theories that disagree on the validity of arguments.

We should be careful, however, not to confuse a logical theory with a formal system. A logical theory is not a proof system or a formal semantics with a consequence relation. Granted, formal systems typically contribute to

\footnotetext{
${ }^{3}$ Quine's case for classical logic has met with criticism elsewhere (e.g. Arnold \& Shapiro 2007, Priest 2006a).
}

Australasian Journal of Logic (16:7) 2019, Article no. 4 
logical theories. Our understanding of the property of validity is improved by formal counterparts such as model-theoretic consequence. But a logical theory should not be equated with the model theory. The model theory is not an account of logical properties in its own right - it is merely a formalism. For it to be part of a theory it requires an application, and it is the logical theory that states what the application is. No one is disagreeing about, say, whether the law of double negation is classically valid. It is, and that is uncontroversial. The disagreement is about whether or not it is genuinely valid. ${ }^{4}$

Nonetheless, for simplicity I will sometimes talk about 'the classical theory', 'the paracomplete theory', and so on. This is a simplification, however, since there is strictly speaking no single classical theory. Roughly, a classical theory will be one that equates genuine validity with classical validity. That is, it accepts the law of excluded middle, Peirce's Law, etc. Similarly, a paracomplete theory is a logical theory according to which the law of excluded middle is not genuinely valid. But the disagreement does not stop with the extension of validity. The nature of validity is equally contentious. Indeed, rival logical theories often do not have the same account of genuine validity. Classicists, relevantists, and intuitionists don't only disagree about the extension of validity, they disagree about what is preserved in valid arguments, about whether or not valid arguments are constructive or have the property of variable-sharing.

It follows, therefore, that two theories can equate validity with, say, classical validity, but nonetheless disagree about other logical properties. For example, two theories may give different accounts of consistency, provability, negation, and so on. For the classicist, negation is truth-functional and bivalent; for the paracompletist it's truth-functional but trivalent; while for the intuitionist it's neither. Similarly, logical theories differ on what the logical form of an argument is, and about which operators qualify as logical (e.g. first-order vs. second-order quantifiers). What is more, there is no agreement on the truth-bearers that constitute an argument. According to some views, logical consequence is a relation on propositions, for others it is sentences, or even utterances. ${ }^{5}$ In other words, both the consequence relation and its relata are up for grabs in logical theories.

What sorts of properties are logical properties? That varies from logical theory to logical theory. This is not the place for an exhaustive taxonomy of

\footnotetext{
${ }^{4}$ The term 'genuine validity' is from Field (2015). Priest (2014) introduces the idea that what the disagreement is about is the 'canonical application' of logical systems, namely the application to deductive reasoning.

${ }^{5}$ See Russell (2008) and Zardini (2014) for more on the relata of logical consequence.
} 
logical theories, but there are some distinctions we will do well to keep in mind. A key divide is between metalinguistic and non-metalinguistic theories. Metalinguistic theories hold that validity is a property of language. There is no shortage of examples: Carnap's (1937) conventionalism about logic, Dummett's (1991) inferentialism, and Shapiro's (2014) logic-as-modelling. In contrast, non-metalinguistic theories claim that validity is ultimately a property of the non-linguistic world. Russell's (1918) logical atomism is an early example, but more recent variants include Williamson (2013b; 2017), Sider (2013), and Maddy (2014). The divide concerns the foundations of validity as a property, but it also raises questions about the relata of valid arguments. Are they linguistic entities (e.g. sentences), or are they Russellian propositions or states of affairs?

Moreover, metalinguistic and non-metalinguistic logical theories are supported by different types of evidence. In virtue of being theories about language, metalinguistic theories are typically supported by semantic considerations. One example, which remains anti-exceptionalist in spirit, is Shapiro (2014). According to Shapiro, logical theories are theories about natural language expressions, their semantics and inferential role. On this picture, rival logical theories offer different semantic models of natural language connectives and quantifiers. Logical theories of this sort are presumably supported by semantic evidence, for instance by eliciting language intuitions from competent speakers. Indeed, Shapiro suggests we apply the methodology directly to expressions such as 'is valid' by testing for covert variables that are fixed by context (ibid., 114-20).

In contrast, semantic evidence is not especially relevant to non-metalinguistic theories. In Williamson's (2017) non-metalinguistic theory, logical claims are ultimately just unrestricted generalizations about the world. Consider a toy example, the validity of the law of excluded middle:

$$
\text { (1) } \vDash \varphi \vee \neg \varphi
$$

The suggestion is that a logical theorem can be expressed as an unrestricted generalization in a higher-order background language:

$$
\text { (2) } \forall X(X \vee \neg X)
$$

The claim in 2 is the universal generalization of 1 , that is, the latter's nonlogical expressions are replaced by appropriate variables. ${ }^{6}$ The schematic claim in 1 is typically weaker than the objectual claim in 2 . The former is

\footnotetext{
${ }^{6}$ See Williamson (2013b, 200).
} 
simply a shorthand for saying that every sentence of the right form is true. In contrast, the latter makes an unrestricted claim about the world, regardless of potential limitations of the language in question. Williamson's point is that 2 is not a metalinguistic claim, but an unrestricted generalization about matters in the world-linguistic or non-linguistic. The analysis anchors claims about validity to strictly non-metalinguistic affairs. What is more, 2 avoids talk of necessity, logical truth, or other modal notions. Both features are among the chief virtues of Tarski's model-theoretic analysis of validity.

It does not matter for my purposes whether validity actually can, or ought to, be expressed by unrestricted generalizations. ${ }^{7}$ The point is just that logical theories may be metalinguistic or non-metalinguistic. What matters is that the choice will be reflected in the evidence required to confirm or disconfirm the theory. I will return to the issue of evidence in detail in Section 3.

A logical theory, then, can be either metalinguistic or non-metalinguistic, depending on its claims about validity. Another important question is whether logical theories are normative or descriptive. The short answer is that on the account I am developing here, logical theories are descriptive. It is true that logic as a discipline contains normative projects. It gives prescriptions about how we ought to reason deductively, it tells us what we may infer, and what we are committed to. Put in epistemological terms, logic is sometimes seen as providing norms of belief. ${ }^{8}$ But logical theories, in the present sense, are not normative. Rather, they describe logical properties by, for example, attributing validity to arguments, or properties such as truth-preservation and variable-sharing to validity. Exactly what is being described-language or the world-will depend on the theory in question. ${ }^{9}$

It will therefore be important to keep logical theories separate from theories of belief-formation, belief-revision, and reasoning. ${ }^{10}$ The latter theories are primarily concerned with norms of rational belief. And although a theory of validity and other logical properties certainly will inform theories about reasoning, and vice versa, that is a downstream consequence. ${ }^{11}$ It is possible to subscribe to the same logical theory, the same account of validity, without

\footnotetext{
${ }^{7}$ There are a number of problems, some of which are discussed in Hjortland (2017).

${ }^{8}$ Alternatively, logic can be seen as governing norms for assertion and denial, or for acceptance and rejection. See for example Restall (2005) and Hjortland (2014).

${ }^{9}$ There is another descriptive issue I'm not discussing here. One type of theory gives a description of how agents actually reason. That is subject matter of theories in the psychology of reasoning.

${ }^{10}$ It is a distinction most successfully promoted by Harman $(1984 ; 1986)$.

${ }^{11}$ See MacFarlane (2004), Field (2009), and Steinberger (2016; 2019) for a number of proposals about the connection between logical properties and norms.
}

Australasian Journal of Logic (16:7) 2019, Article no. 4 
therefore also agreeing about the normative status of logic. Two logicians who both support the classical theory of consistency, for example, might disagree about the normative upshot for inconsistent belief sets, say, because of the Preface Paradox. Conversely, two distinct logical theories may be compatible with a single theory of inference and belief revision.

\section{Abductivism and evidence}

There is a wide variety of claims a logical theory can make, and hence a wide variety of claims that require evidential support. When we now turn to evidential confirmation of logical theories, however, the main concern will not be the individual claims but entire theories. In what follows I will assume that some form of abductivism for logical theories is correct. Rival logical theories joust for position by better accommodating the evidence.

A number of philosophers have recently supported abductivism. Russell $(2015,8)$, for example, gives an account of how we come to justify our logical beliefs. Her main point is that reasoning, and specifically inference to the best explanation, is more basic than logical theories:

The simplicity, elegance, fertility, low-cost, and explanatory power of an entire logical theory played a part in the [...] reasons for believing or not believing it and its parts. [...] Once we got beyond very early pre-theoretic reasoning, logical laws like the law of excluded middle were given up or adopted as a part of an entire logical theory.

There are two things we should note. First, confirmational holism is part of the account. A logical theory is confirmed en bloc, not by individual confirmation of logical laws. Second, Russell lists a number of theoretical virtues that will help us choose between candidate logical theories. These virtues are all familiar from theory choice in general philosophy of science, and they are also endorsed by other anti-exceptionalists about logic. Here is Priest (2014):

Given any theory, in science, metaphysics, ethics, logic, or anything else, we choose the theory which best meets those criteria which determine a good theory. Principal amongst these is adequacy to the data for which the theory is meant to account. In the present case, these are those particular inferences that strike us as correct or incorrect. This does not mean that a theory 
which is good in other respects cannot overturn aberrant data. As is well recognised in the philosophy of science, all things are fallible: both theory and data. Adequacy to the data is only one criterion, however. Others that are frequently invoked are: simplicity, non-(ad hocness), unifying power, fruitfulness. (Priest 2014, 217)

The virtues suggested by Priest are to a large extent the same as Russell's, but his emphasis is on adequacy to the data.

Finally, Williamson $(2017,14)$ echoes the same list of theoretical virtues, insisting that they are part of an abductive methodology:

[S]cientific theory choice follows a broadly abductive methodology. [...] Scientific theories are compared with respect to how well they fit the evidence, of course, but also with respect to virtues such as strength, simplicity, elegance, and unifying power. We may speak loosely of inference to the best explanation, although in the case of logical theorems we do not mean specifically causal explanation, but rather a wider process of bringing our miscellaneous information under generalizations that unify it in illuminating ways.

Despite the apparent agreement about which selection criteria apply, it is clear that the criteria need further explanation. Their application to logical theories will most likely differ from the application to non-logical theories. Furthermore, the three authors apply the criteria to reach very different conclusions about the correct logical theory. In order to have any practical impact on the choice of logical theories, therefore, the criteria need careful articulation. In addition, they should be assigned a weight that indicates how they affect the theory-choice.

I have already discussed some of the selection criteria in detail elsewhere (Hjortland 2017). In what follows my aim will be more limited. The main concern is to identify what counts as evidential confirmation of logical theories. The motivation is that 'fit with the data' is arguably the most important of the theoretical virtues associated with abduction. (In other words, it is assigned the greatest weight.) After all, in inference to the best explanation, something has to be explained. That something is the evidence. A theory can explain the evidence better than another, either by more fully accounting for the evidence, or by offering, say, a simpler or more unified explanation. The issue of evidential confirmation is at the heart of abductivism. It is 
frustrating, therefore, that there is no agreed upon account of what counts as evidence for a logical theory.

\section{Intuitions about validity}

\subsection{General remarks}

Let us start out by looking at what the above authors say about evidence for logical theories. First consider Priest:

One of the criteria may give pause, however. In the criterion of adequacy to the data, what counts as data? It is clear enough what provides the data in the case of an empirical science: observation and experiment. What plays this role in logic? The answer, I take it, is our intuitions about the validity or otherwise of vernacular inferences. (Priest 2016, 9)

Priest is not the only one who thinks that intuitions play a central role in the evaluation of logical theories. Russell (2015, 5-6) suggests that, although the logical theory consists of theoretical concepts, it explains, unifies, predicts, and regiments pre-theoretic intuitions: ${ }^{12}$

If you start with some intuitions and a few inchoate pre-theoretical dictums, a theory with the virtues of classical logic is an enormous leap forward. The virtues include: explanation, unification, prediction, regimentation: classical logic explains what is special about sentences like $\varphi \vee \neg \varphi$, but also, in a similar way, what is special about other schemata, such as $\varphi \rightarrow \varphi$, and $\neg(\varphi \wedge \neg \varphi)$. The theory predicts that they will be special, they are logical truths. It also gives us results in cases where we may have had no clear intuitions beforehand, such as with Pierce's law: $((\varphi \rightarrow \psi) \rightarrow \varphi) \rightarrow \varphi$. Sometimes the theory predicts results which the student may initially find counterintuitive, such as $(\varphi \rightarrow \psi) \rightarrow(\neg \psi \rightarrow \neg \varphi)$ but further reflection shows it to have been correct. In addition to explaining the presence of the property of logical truth, the theory explains and predicts the presence of other related properties, such as consequence, inconsistency and equivalence. And moreover the same, minimalist basis does the work in all of these different cases. That is elegant.

\footnotetext{
${ }^{12}$ In the example, Russell is talking about a student learning classical logic, but it is clearly meant as a general observation about supporting and revising logical theories.
}

Australasian Journal of Logic (16:7) 2019, Article no. 4 
A logical theory, then, is a candidate explanation of a set of intuititions about the validity or invalidity of arguments in natural language. Of course, neither Russell nor Priest is saying that intuitions are the only available evidence (more on that below), but it is part of the evidence that a logical theory answers to.

In order to show us what intuitions about validity are like, Priest (2016) asks us to consider particular instances of arguments:

If Napoleon is in Strasbourg, he is in France.

Napoleon is in Strasbourg.

Therefore, he is in France.

What sort of intuition do we have about the argument? Priest claims that arguments like this 'strike us as correct' (ibid.). Correspondingly, other arguments will presumably strike us as incorrect. For example:

If Napoleon is in Strasbourg, there is war.

There is war.

Therefore, Napoleon is in Strasbourg.

Suppose you already believe the premises of the first argument. Then you may very well have the intuition that Napoleon is in France prior to reflection. Yet, that is not an intuition about the validity of the argument, but an intuition about its conclusion. Indeed, maybe you will infer that Napoleon is in France, and hence form the outright belief. But that belief is neither here nor there with regard to the validity of the argument. Maybe the intuition about the conclusion is the result of a triggered disposition to infer according to Modus Ponens. However, having a disposition to infer is not the same as having an intuition about the correctness of an argument.

Do we in addition have an intuition about the correctness of the argument itself? Suppose you have no opinion about the premises. In that case, maybe there is nonetheless an alethic intution that the argument preserves truth. Alternatively, there could be an epistemic intuition to the effect that one ought not believe the premises and disbelieve the conclusion simultaneously. I am not going to argue against the existence of such 'correctness' intuitions. If they do exist, however, there is still the question of their status as evidence. There are some reasons to have reservations about how much evidential work intuitions can do.

First, if the intuition is merely that the argument is truth-preserving, there is not sufficient evidence to conclude that the argument is necessarily truth-preserving. That is, the intuition better have a modal component to 
separate it from the intuition that an indicative conditional is true. Similarly, an intuition that one ought not to believe the premises and disbelieve the conclusion could attach to the specific content of the premises and the conclusion. In that case, the intuition presumably won't support the claim that the argument is valid. The intuitions would have to be of a different form, for example, that it cannot (pause for modal force) be that the premises are true and the conclusion false. In epistemic terms, it is irrational-and so not merely inaccurate - to believe the premises and disbelieve the conclusion.

Second, the intuition about the argument above is an intuition about an instance of Modus Ponens. But a logical theory doesn't make claims about instances of Modus Ponens. A theory that endorses Modus Ponens claims that all arguments of a certain form are valid. The problem is how we bridge the gap between intuitions attached to a limited pool of instances and the evidential task of confirming a universal claim. In contrast, intuitions about the second argument are more straightforward. In order to count the argument as invalid, all that is needed is a single counter-example, although what constitutes a counter-example will differ from theory to theory. The burden of evidence for validity appears greater. Attributing validity to an argument is making a universal claim to the effect that there is no counterexample. Priest is aware of the problem, and cautions that intutions about argument correctness may be due to an 'impoverished diet of examples' (ibid., 10). I agree. That doesn't mean that intuitions cannot be evidence, but the strength of the evidence is limited.

Third, the source of the intuitions is a further worry. What are the options? The intuitions could emanate from a rational faculty (e.g. Gödelian intuition), but that doesn't sit very well with the nonapriorist commitments of anti-exceptionalism. Another option is that the intuitions are the result of ordinary language users' understanding of logical expressions (e.g. 'not', 'if ...then', 'therefore'). The anti-exceptionalist can accept that there is such a connection between understanding and intuitions without therefore thinking that it gives an a priori justification. The semantic intuitions merely provide defeasible evidence. The nonapriorist can even concede that the intuitions are a result of analytic truths. It does not entail that the analyticity is sufficient to provide knowledge, justified belief or even entitlement. ${ }^{13}$

If the intuitions are semantic, however, that leads to other difficulties. It might sound promising that the intuitions attach to an understanding of logical expressions in natural language, but the connection between logical theories and natural language expressions is easy to exaggerate. At best,

${ }^{13}$ See Russell (2014) and Williamson (2007, chs. 3-4).

Australasian Journal of Logic (16:7) 2019, Article no. 4 
logical theories account for highly idealized features of natural language expressions, far removed from the many frictions of empirical linguistics. Glanzberg (2015), for example, argues that a theory of the semantics and syntax of natural language expressions does not provide an account of validity or other logical properties. Why then should we assume that just because evidence helps to uncover the syntax and semantics of natural language, it is equally helpful with respect to logical properties?

More importantly, why should we think that our natural language expressions are a good guide to logical properties? Like all scientific theories, logical theories sometimes introduce new concepts that replace old ones for theoretical reasons. ${ }^{14}$ Consider what happens in non-logical theories. An ordinary expression 'mass' or 'star' is replaced by increasingly sophisticated and formalized concepts, which are better suited to the explanatory task. As a result, relying on semantic intuitions about the defunct concept will lead one astray. Logical theories are no different. ${ }^{15}$ The concepts most logical theories trade in are not pre-theoretical concepts, but at best explications of natural language counterparts. This is especially evident for theoretical concepts such as validity, consistency, and countermodel, but even concepts such as conditional, negation, and truth bear only stylized resemblance to natural language counterparts. Understanding natural language expressions, therefore, is no fool-proof guide to logical properties.

\subsection{Intuitions and classicism}

These more general concerns aside, do intuitions provide evidential support for the classical theory? The situation is not clear-cut. On the one hand, it is a strength of the classical theory that it does a decent job systematizing intuitions about arguments. On the other hand, many of the most persistent objections to classical logic are based on purported counterexamples where intuitions differ. McGee's counterexample to Modus Ponens, the paradoxes of the material implication, and examples involving vague expressions have all been used to argue against the classical theory. But there are droves of other examples, some of which are catalogued by Priest (2008).

If we look beyond the intuitions of the experts, the intuitions look even less promising. In fact, what we know from the psychology of reasoning gives us reason to believe that intuitions depart significantly from classical logic.

\footnotetext{
${ }^{14}$ See Scharp (2013) for discussion.

${ }^{15}$ Priest (2006a, 169) makes a similar comparison when discussing Quine's meaningvariance argument. He also subscribes to the view that logical theories trade in technical concepts (ibid., 170).
}

Australasian Journal of Logic (16:7) 2019, Article no. 4 
When agents' judgements about arguments have been tested experimentally, the results suggest that the judgements are influenced by a range of nonlogical factors. For example, judgements about instances of Modus Tollens vary significantly depending on the subject matter. ${ }^{16}$ Should we count this as evidence against Modus Tollens? Other experiments show a systematic belief bias. Subjects are more likely to accept an argument if they already believe the conclusion. ${ }^{17} \mathrm{~A}$ better approach is to insist, following Priest, that intuitions are highly defeasible evidence. Theoretical considerations might count against the data provided by intuitions, and ultimately tip the balance (see Section 5).

Furthermore, it is not clear that intuitions help when it matters. Suppose there are indeed some argument forms for which we have stable intuitions about correctness. These arguments tend not to be the most contentious. The controversial arguments are harder to solicit stable intuitions for (e.g. explosion, classical reductio ad absurdum, Peirce's Law). This suggests that intuitions might provide evidence for logical theories, but that they will not be of much help in singling out the classical theory. They are simply not sufficiently fine-grained to distinguish between the major rival theories. That concern becomes all the more urgent when we recall that a logical theory not only provides an extension for validity, but an account of the nature of validity. Even if the intuitions are evidence towards which arguments are valid, they are hardly evidence for an account of validity itself.

\section{The Quine-Williamson account}

\subsection{General remarks}

Evidence for logical theories is not restricted to intuitions. Fortunately, as Russell and Priest both stress, intuitions are commonly overridden by other considerations. Generally speaking the logical theory is shaped by our confidence in other claims, for example the claims of our best non-logical theories. That should be no surprise when we consider that non-logical theories need not be primarily empirical. We know from ongoing debates that rival views of logic are the result of theories about vagueness, theories about truth, theories about properties, etc. In general, semantic paradoxes that challenge the logical orthodoxy are a particularly rich source of evidence.

\footnotetext{
${ }^{16} \mathrm{~A}$ famous example is the Wason Selection Task (cf. Wason 1966). A number of authors have claimed to find content-sensitivity in subsequent experiments (e.g. Wason \& Shapiro 1971, Griggs \& Cox 1982).

${ }^{17}$ See for example Evans et al. (1983), Morley et al. (2004).
}

Australasian Journal of Logic (16:7) 2019, Article no. 4 
Priest (2006a; 2006b), for example, takes theories of truth to be a decisive source of evidence in favour of a paraconsistent theory. Russell (2015) uses vague expression as an example of the sort of consideration that might lead one to abandon a classical theory.

In contemporary debates, revisionary arguments have been promoted for a number of theories, from quantum mechanics to vagueness. But exactly how do non-logical theories help confirm or disconfirm a logical theory? I want to look more closely at a specific suggestion that has received some attention. It is a view that has been attributed to Quine (e.g. Wright 1986, Field 2000, Boghossian 2000). ${ }^{18}$

Here is Field's formulation:

Quine's view is that one should evaluate alternative logics in combination with theories of the rest of the world: given a theory of everything, including a logic, one uses the logic in the theory to generate the theory's consequences. Then we choose a theory, including a logic, on such grounds as overall simplicity and conservativeness and agreement with observations. (Field 2000, 129)

Boghossian gives a similar, although somewhat more elaborate sketch:

According to [Quine's] account, warrant accrues to a logical principle in the same way that it accrues to any other empirical belief, by that principle's playing an appropriate role in an overall explanatory and predictive theory that maximizes simplicity and minimizes the occurrence of recalcitrant experience. We start with a particular theory $T$ with its underlying logic $L$ and from $T$ we derive, using $L$, a claim $p$. Next, suppose we undergo a string of experiences that are recalcitrant in that they incline us to assent to not- $p$. We need to consider how $T$ might best be modified in order to accommodate this recalcitrance, where it is understood that one of our options is to so modify the underlying logic of $T$ that the offending claim $p$ is no longer derivable from it. We need to consider, that is, various ordered pairs of theory and logic - $<T, L>,<T^{\prime}, L>,<T^{\prime \prime}, L>\ldots,<T, L^{\prime}>$, $<T, L^{\prime \prime}>\ldots$ - picking that pair that entails the best set of observation sentences. Whatever logic ends up being so selected is the logic that is maximally justified by experience. In rough

\footnotetext{
${ }^{18}$ As far as I know, Quine does not formulate the view in detail, although it is a plausible interpretation of Quine (1951).
}

Australasian Journal of Logic (16:7) 2019, Article no. 4 
schematic outline, that is the Quinean picture. (Boghossian 2000, 233)

The account has found a more recent champion in Williamson (2017), and since it is his formulation I will go on to discuss, I call it the 'Quine-Williamson account'. Suppose that we have two rival consequence operators $\models_{1}$ and $\models_{2}$. Let $\Gamma$ be a set of sentences - we will call it the antecedent theory. Consider the closures of $\Gamma$ under $\models_{1}$ and $\models_{2}: C n_{\models_{1}}(\Gamma)$ and $C n_{\models_{2}}(\Gamma)$.

Suppose that we are comparing the consequence relations $\models_{1}$ and $\models_{2}$. Then we should not simply compare the theorems of $\models_{1}$ with the theorems of $\models_{2}$. Rather, we should compare the theories they generate from independently well-confirmed sentences, such as well-established principles of physics. That is, we should compare $C n_{\models 1}(\Gamma)$ with $C n_{\models 2}(\Gamma)$ as theories for various independently well-confirmed sets $\Gamma$ of sentences of $L$. We require $\Gamma$ to be highly confirmed because the best of logics will draw some bad conclusions from bad premises, and for reasons of methodological fairness we require the confirmation to be independent in the sense that it is not too sensitive to the choice of logic. Comparing the theorems of the consequence relations is just the limiting case where $\Gamma=\{\}$, for the empty set is vacuously well-confirmed. (Williamson 2017, 13-4)

One of Williamson's motivations is that the account of evidence for logical theories is non-metalinguistic. ${ }^{19}$ Given that the antecedent theory $\Gamma$ is non-metalinguistic (e.g. a theory of physics), the closure $C n_{\models_{i}}(\Gamma)(i \in$ $\{1,2\})$ is also non-metalinguistic. The assessment of $C n_{\models}(\Gamma)$, therefore, relies primarily on non-metalinguistic evidence. The sentences in $\Gamma$ are not specifically about validity or other logical properties. They are run of the mill claims about the world. When we compare the two output theories, then, we are comparing them with respect to evidence about non-metalinguistic states of affairs. The result is indirect confirmation or disconfirmation of logical theories, without logic-specific evidence.

Suppose that we start with a well-confirmed antecedent theory $\Gamma$ and a consequence relation $\models .{ }^{20}$ The members of $\Gamma$ could for instance be generalizations or laws from physics, chemistry, or economics. What happens if there is

\footnotetext{
${ }^{19}$ Recall the distinction between metalinguistic and non-metalinguistic theories in Section 2 .

${ }^{20}$ It might be problematic to require that $\Gamma$ be 'highly confirmed' (see the quote from Williamson above). We want to know what follows logically from an explicitly inconsistent
} 
a sentence $A \in C n_{\models}(\Gamma)$ that is independently disconfirmed by evidence? That leads to a conflict: The direct evidence tells against $A$, but the antecedent theory $\Gamma$ closed under $\models$ tells in favour of it. There are different ways of resolving the tension. Depending on the strength of the evidence in question, we might either maintain $A$ despite the evidence, or give up some member of $\Gamma$ such that the revised theory $\Gamma^{\prime}$ does not have $A$ in its $\models$-closure. The third option is the one that concerns logical theories. We can give up the consequence relation $\models$, or, more precisely, give up the relevant claims of the logical theory that provided $\models$. Instead we close the theory $\Gamma$ under a consequence operator $C n_{\models^{\prime}}$ that does not produce $A$.

Let us consider an example in some more detail. Suppose you have two rival consequence operators, $C n_{\models_{1}}$ and $C n_{\models_{2}}$, the first of which satisfies disjunctive syllogism (i.e. $A \vee B, \neg A \models_{1} B$ ), the other not. What constitutes evidence for and against a theory that holds disjunctive syllogism to be valid? Presumably, any account of validity will require that in order for disjunctive syllogism to be valid, it has to be the case that whenever $A \vee B, \neg A \in \Gamma$, $B \in C n_{\models}(\Gamma)$. So let $\Gamma$ be a well-confirmed antecedent theory, and suppose, for some particular sentences $p, q: p \vee q, \neg p \in \Gamma$. If it turns out that $q$ is an independently disconfirmed sentence, then it is tempting to conclude that there is evidence against the validity of disjunctive syllogism. ${ }^{21} \mathrm{How}$ suggestive is that evidence? It is by no means conclusive. There is still the possibility that despite its plausibility, the antecedent theory $\Gamma$ ought to be overturned. Alternatively, it may be that the evidence against $q$ has been misleading. In other words, rejecting the validity of disjunctive syllogism, or any other argument form, is no mean feat.

On the other hand, the evidential burden is even greater when we want to include the validity of an argument form. Suppose again that $\Gamma$ is a well-confirmed antecedent theory. First, it would be too stringent to require that whenever $A \vee B, \neg A \in \Gamma, B$ is well-confirmed. In many cases there will be no evidence either way for $B$, and that shouldn't necessarily be evidence against the validity of the argument form. (For many sentences, the only available evidence is that they are logical consequences of well-confirmed sentences.) In the unlikely event that whenever $A \vee B, \neg A \in \Gamma, B$ is not disconfirmed, we have something close to the optimal evidential support. Yet,

theory. In fact, the law of explosion is a highly contentious logical law. And even if a subtly inconsistent theory might be highly confirmed, an explicitly inconsistent theory is unlikely to be.

${ }^{21}$ Note that it is unfortunate to insist that $\neg q$ is well-confirmed, given that for some logical theories there will sentences $A$ such that $A$ and $\neg A$ are both well-confirmed (e.g. because $A$ is paradoxical and therefore both true and false).

Australasian Journal of Logic (16:7) 2019, Article no. 4 
that is still only relative to a particular antecedent theory $\Gamma$. More generally, we would like it to be the case that, for every well-confirmed antecedent theory $\Gamma$, whenever $A \vee B, \neg A \in \Gamma, B$ is not disconfirmed. It is even better if $B$ subsequently receives independent confirmation, but that cannot always be expected. Instead it may be that future evidence tells against $B$, in which case we are back in the old predicament: reject $\Gamma$ or reject the consequence relation.

\subsection{The Quine-Williamson account and classicism}

According to Williamson, this account of evidence supports the classical logical theory. It provides the evidential confirmation that ultimately fuels an abductive argument for classical logic. Other factors such as strength and simplicity also play a role, but they are presumably secondary. (We will revisit them in Section 7.) The question, then, is why we should think that a classical theory does particularly well given the Quine-Williamson account. Let us start by looking at some concrete cases.

Mathematics: One case that is typically thought to be favourable to the classical theory is mathematics. Let $\Gamma$ be the Peano axioms of arithmetic, and $C n_{\models} C L$ the first-order classical consequence operator. Then Peano Arithmetic (PA) is given by $C n_{\models_{C L}}(\Gamma)$. It is hard to deny that the classical formalization of arithmetic is a success story. Classical logic has provided an illuminating regimentation of informal proofs, one that captures the practice of working mathematicians and assists the in search for new theorems and new proofs. The classical consequence operator is, in other words, supported by the arithmetic theory. It delivers no dubious conclusions, nor do we have an alternative consequence operator that delivers better results. Worse, combining arithmetic with nonclassical logics undercuts powerful results. Even if nonclassical arithmetics are underexplored, it seems to count against them that they have generated only limited mathematical interest. ${ }^{22}$ True, there remains controversial questions, such as whether we should prefer a second-order consequence relation to a first-order, but nothing that threatens to undermine the choice of classical logic in mathematics.

So mathematical theories provide evidential confirmation for the classical theory. That should be no surprise, since classical logic was developed precisely in order to formalize mathematical proof. If it had not been suited

\footnotetext{
${ }^{22}$ They do deserve better, however. See Weber $(2010,2012)$ for some honest toil in nonclassical mathematics. See also Shapiro (2014) about why we should be pluralists about logic in mathematics.
}

Australasian Journal of Logic (16:7) 2019, Article no. 4 
for that purpose, it would not have had such an auspicious start. Rather, it is the unparalleled success of classical logic in mathematics that has led to its canonization, and in turn to its application beyond mathematics. The question is not whether classical logic is a good fit with mathematics, but whether it is wise to conclude more ambitiously that classical logic fits all purposes. Since it is favoured by mathematics, the classical theory already has a head start over its competitors. To the extent that mathematics is involved in other theories (e.g. physics, chemistry, economics), classical logic also appears to be a reasonable companion to our best theories. We will look closer at the argument from mathematics in Section 6.

QUANTUM MECHANICS: What, then, can be said against the classical theory? In order to answer that, let us first consider what would constitute evidence against the classical theory. A possible scenario would be that an empirically well-confirmed theory cannot be closed under classical consequence without resulting in independently unlikely conclusions. The closest we have been to that is the potential revision of classical logic in favour of quantum logic. Following an observation by Birkhoff \& von Neumann (1937), Putnam (1969) argued that '[w]e live in a world with a non-classical logic.' That is a dramatic conclusion, and one that Putnam first modifies, and then rejects (cf. Putnam 1981, 2012). Even if quantum logic has gone out of favour, however, it serves as an apt illustration of how the Quine-Williamson account in principle could lead us to reject the classical theory.

In classical mechanics, physical events are represented mathematically by subsets of phase spaces, i.e. coordinates for position and momentum. The physical events together with set-theoretic operations yield a Boolean algebra. If we think of the physical events as propositions, and, correspondingly, the set-theoretic operations as logical connectives, classical mechanics is governed by classical logic. In quantum mechanics, however, the mathematical representation of propositions as subspaces of a Hilbert space gives rise to a non-distributive lattice. With the meet, join, and orthocomplement operators interpreted as logical connectives, the result is a logic where the law of distributivity fails (i.e. $A \wedge(B \vee C) \not \models(A \wedge B) \vee(A \wedge C))$-more precisely, quantum logic.

Putnam's (1969) methodology is precisely the one suggested by Williamson, although Williamson of course rejects the revisionary conclusion. Putnam argues that if quantum mechanics is closed under classical logic, the result is conclusions that are indefensible - in particular, that the measurements 'disturb the very magnitude they seek to measure' (ibid., 183). What is worse, the parts of quantum mechanics that deliver the problematic conclusions are 
experimentally well-confirmed. The rest is a cost-benefit analysis. Putnam claims that classical logic ought to be abandoned in favour of quantum logic, which purportedly does not produce the same problematic conclusions.

Putnam's revisionary argument has been discredited for a number of reasons (cf. Maudlin 2005), but the debate seems to partly vindicate the proposed methodology of the Quine-Williamson account. If a well-confirmed theory delivers unpalatable conclusions when closed under a consequence operator, that counts as evidence against the consequence operator. In the case of quantum logic, the sticking point was whether we would rather live with the conclusions or give up classical logic. The subsequent development has sided resoundingly with classical logic.

SEMANTIC PARAdOXEs: It is telling that the most fertile ground for revisionary arguments is not the empirical claims of natural sciences. It is, rather, philosophical theories. The standard case against classical logic is not quantum mechanics or anything in the neighbourhood, but the semantic paradoxes. The reason, I suspect, is that in the case of the semantic paradoxes the problem is not only that classical logic yields evidentially unlikely consequences, but that it is simply inconsistent with desirable non-logical claims. A familiar example is formal theories of truth. Suppose $\Gamma$ is the Peano axioms together with the unrestricted $T$-schema (i.e. $T\left({ }^{\ulcorner} A^{\urcorner}\right) \leftrightarrow A$ ). The closure $C n_{C L}(\Gamma)$ is trivial since the set $\Gamma$ is classically inconsistent.

Recall that in the quantum logic case, the theorist could hold on to the experimentally supported quantum mechanics claims in $\Gamma$, and, perhaps hesitatingly, accept the classical conclusions. They might be unlovely conclusions from a theoretical perspective, but they can be accommodated as part of a cost-benefit analysis. On the other hand, the deflationist about truth who is committed to the unrestricted $T$-schema cannot simply bite the bullet. Unless one is willing to embrace trivialism, a fringe position to say the least, classical logic has to go. A similar dynamics can be found in theories about vague expressions. Tolerance principles for vague expressions lead to triviality when closed under classical consequence. Unless one is willing to drop the tolerance principles, classical logic simply isn't an option. This explains why Williamson $(2017,21)$ calls the semantic paradoxes the best case for revision of logic. Although he thinks we should ultimately resist the revision, he does acknowledge that the revisionary arguments need detailed attention. The semantic paradoxes provide crucial evidence that any abductive argument must account for.

Even if the Quine-Williamson account appears to capture the dialectics of Australasian Journal of Logic (16:7) 2019, Article no. 4 
at least some revisionary debates, it gives no conclusive advantage to the classicist. The nonclassicist holds that there are semantic theories that the classical theory cannot accommodate on pain of triviality. The classicist is free to reject the antecedent semantic theories to save her logical theory, but that comes with a cost. Likewise, since the nonclassicist rejects classical principles, she must provide an alternative account of mathematical proof. That will either involve a revisionist account of mathematics (e.g. intuitionism), or a recapture argument to the effect that the nonclassical theory is compatible with local classical principles for mathematical theories. Both strategies have shortcomings - more about that in Section $6 .^{23}$

Another concern with the Williamson-Quine account is that it might carry a classical bias. Suppose we think that truth preservation is a necessary requirement for validity. In that case, confirmed premises and a disconfirmed conclusion constitute evidence against the validity of the argument. But, a number of nonclassical theories claim that truth-preservation is a necessary condition for valid arguments, but not a sufficient condition. According to some of these theories, valid arguments preserve another property. Validity is sometimes taken to preserve relevance, semantic content, information, or warrant. If you think validity is a matter of truth preservation plus another property, there is no reason to think that evidence against the validity of an argument need be confirmation of the premises and disconfirmation of the conclusion. For there is nothing preventing an invalid argument from necessarily preserving truth.

Let us consider some examples that highlight the problem. First, suppose we have a scenario with two rival logical theories, both of which claim that truth preservation is necessary and sufficient for validity. Suppose further that the first theory says that Modus Ponens is valid, and the second says that it's not. If there is an instance of Modus Ponens where the premises are confirmed, and yet the conclusion disconfirmed, that counts against the former theory. We might, for example, think that McGee's (1985) purported counterexample is an example of the type of evidence that is pertinent to the dispute. (Although few would discard Modus Ponens on the basis of the example.)

In the first scenario, the Quine-Williamson account presumably works as intended. Now let us consider instead a scenario with two rival theories where only the first claims that truth preservation is necessary and sufficient for validity. The second theory claims that truth preservation is necessary for validity, but it also requires another property. Moreover, the first theory

\footnotetext{
${ }^{23}$ See also Woods (2019) for an insightful discussion of the recapture strategy.
} 
claims that the law of explosion (i.e. $A, \neg A \models B$ ) is valid, the other rejects it. Finally, both theories hold that the law of explosion preserves truth. What evidence can the Quine-Williamson account offer to distinguish the two theories? Since both theories claim that the law of explosion is truthpreserving, confirmation of the premises and disconfirmation of the conclusion would be counter-evidence. But, confirmation of the premises is in any case highly implausible. It is more likely that no counter-evidence is forthcoming. In fact, it is likely that in most cases the premises will be disconfirmed. According to the account, this is good news for a logical theory that claims the law of explosion is valid. But that is misleading at best. The second theory agrees that the argument preserves truth, and therefore gives grounds to expect the same pattern of confirmation and disconfirmation as the the first theory. It does not, however, claim that the argument is valid simply because it is truth-preserving.

The Quine-Williamson account is supposed to offer evidence that assists the choice between rival logical theories. But not all logical theories equate validity with truth preservation. While the preservation of truth might be supported by patterns of confirmation and disconfirmation, other properties of valid arguments need not be similarly supported by the evidence afforded by the Quine-Wiliamson account. Hence, even if the Quine-Williamson account delivers credible evidence for or against logical theories, the evidence cannot settle key disputes about the choice of logical theory.

\section{Indispensability arguments}

\subsection{General remarks}

The Putnam-Quine indispensability argument is a familiar naturalist defence of realism for mathematics. Given the close connections between mathematics and logic, it is seems sensible for an anti-exceptionalist to apply an indispensability argument for logical theories as well. But despite the amount of attention indispensability arguments have received in the philosophy of mathematics, there is hardly any literature on indispensability arguments for logic. ${ }^{24}$ One explanation is that since logical theories don't trade explicitly

\footnotetext{
${ }^{24}$ An exception is Putnam (1971). Enoch \& Schechter $(2006 ; 2008)$ also argue that certain logical inferences are indispensable for the success of projects we are rationally required to engage in. As a result, agents are justified in applying the inferences in question. Their proposal certainly has merit, but it is orthogonal to the present discussion. I am not looking for an indispensability argument for what Enoch and Schechter call 'basic belief-forming methods', but for logical theories as described in Section 2. Even if certain
} 
in ontology, indispensability arguments are inappropriate.

That is a mistake. Although the Putnam-Quine indispensability argument has an ontological conclusion, it is mediated through an epistemological assumption: since mathematical claims are indispensable to our best theories, the mathematical claims receive indirect evidential confirmation. Field (1989, 14), for example, says that an indispensability argument 'is an argument that we should believe a certain claim (for instance, a claim asserting the existence of a certain kind of entity) because doing so is indispensable for certain purposes (which the argument then details)'.

Is there a plausible indispensability argument for logic? In fact, there is a common argument in favour of classical logic that can be formulated as an indispensability argument. The key components are as follows:

(Premise 1) Mathematics is indispensable to our best theories.

(Premise 2) Classical logic is indispensable to mathematics.

(Conclusion) Therefore, classical logic is indispensable to our best theories.

I am simply going to grant Premise 1, and assume that there is some wellconfirmed theory, say, in physics, for which mathematics is indespensable. I am also going to assume, for the sake of argument, that indispensability does indeed incur evidential confirmation. That is certainly contentious, but there is plenty of discussion of that elsewhere, and I don't have anything new to add to the debate. ${ }^{25}$

\subsection{Indispensability and classicism}

What I would like to focus on is Premise 2. Is it true that classical logic is indispensable to mathematics? As I pointed out in Section 5, there is a strong connection between classical logic and mathematics. It is hard to deny that there are informal mathematical proofs that require instances of characteristically classical arguments (e.g. classical reductio ad absurdum). Without these argumentative steps the power of mathematical proof would be severely curtailed. However, I do not think that the presence of classical arguments in mathematics is sufficient to say that the classical logical theory is indispensable to mathematics.

inferences (e.g. inference to the best explanation) are indispensable epistemic tools in valuable projects, it does not follow that the claims of logical theories are indispensable to our best non-logical theories.

${ }^{25}$ See Colyvan (2001) for a discussion and further references. 
Recall that the classical theory makes claims about validity. For example:

(3) The law of excluded middle is valid;

or

(4) Classical reductio absurdum is valid.

In the classical theory, 3 and 4 are universal in the sense that any instance of the argument forms in question is valid. In other words, the claims are stronger than what is required for mathematical proofs. For it is only certain instances of these argument forms that are required for mathematical proofs, in particular, the instances that concern mathematical properties. And there is nothing preventing a nonclassical theory from incorporating instances of classical principles, without therefore accepting classical validity wholesale.

Let us look at a simple example to shed some light on the possibility. Advocates of an unrestricted truth predicate reject the classical theory, but at least some of them want to simultaneously preserve classical mathematics (Kripke 1975, Field 2008, Horsten 2011). This is done in a number of ways. A well-known example is paracomplete theories of truth built on an arithmetic language supplemented with the truth predicate. In these nonclassical theories, claims such as 3 and 4 are rejected (together with a host of other classical principles). But the theories nonetheless preserve classical mathematics. In model-theoretic terms, the fixed-point models of paracomplete theories are classical for the arithmetic language: no arithmetic sentence receives a nonclassical truth value. Importantly, the logical concepts of the paracomplete theories behave classically for sentences with classical truth values. As a result, the classical principles such the law of excluded middle are recaptured for arithmetic sentences and arguments. Of course, the presence of the truth predicate will complicate matters, but for good reason. The theory would be inconsistent if non-arithmetic sentences behaved classically. For the record, it is not only paracomplete theories that have this recapture property, but a number of nonclassical theories of truth (e.g. paraconsistent theories, substructural theories).

The classical recapture can be viewed proof-theoretically as well. For axiomatizations of paracomplete theories, classical reductio and the law of excluded middle will be admissible given that the language is arithmetical. ${ }^{26}$ As we should expect, if the truth predicate is allowed in to the language, the admissibility fails. Again, the point is that the classical arguments only hold

\footnotetext{
${ }^{26}$ See Halbach \& Horsten $(2006,694)$. See also Restall (1994) for an example from relevant logic.
}

Australasian Journal of Logic (16:7) 2019, Article no. 4 
in special cases. It just happens that they are the special cases that matter for mathematical proofs.

If one prefers to account for validity in terms of unrestricted generalizations (cf. Section 2), there is another option for introducing special cases. If you hold a paracomplete theory, you will reject the validity of the law of excluded middle. That is, you deny the unrestricted generalization 2 that we saw above:

(2) $\forall X(X \vee \neg X)$

Yet, there is in principle nothing preventing the paracomplete theorist from endorsing corresponding restricted generalizations. For example, let $\Theta$ the class of mathematical propositions. Then the following is a special case underpinning the application of the law of excluded middle for mathematics:

(2) $\forall X \in \Theta(X \vee \neg X)$

This might of course be an unwelcome complication of an otherwise simple theory. But the point is not to argue that theories involving classical recapture are superior to the classical alternative. It is merely to argue that the classical theory isn't indispensable to mathematics. That is compatible with there being reasons against the classical recapture that will ultimately lead us to prefer the classical theory.

Maybe there is another strategy for claiming the indispensability of classical logic. Mathematical proofs and mathematical theories rely on the use of classical concepts of negation, implication, and quantification. ${ }^{27}$ That suggests a variation of the indispensability argument. Classical logical concepts are indispensable to mathematics. It is certainly true that formal mathematical theories such as Peano Arithmetic and ZFC are formulated with the help of classical concepts. Even if these formal theories do not directly contribute to other scientific theories, they are nonetheless the most accurate formalization of mathematical concepts and theorems that do.

But just like nonclassical theories can recapture classical validity in special cases, nonclassical logical concepts can recapture classical concepts in special cases. In fact, nonclassical concepts are frequently generalizations of classical

\footnotetext{
${ }^{27}$ Williamson $(2017,22)$ uses this observation as an argument in favour of classical logic, although he does not claim that it makes classical logic indispensable: 'For any complex scientific theory, especially one that involves some mathematics, will make heavy use of negation, conjunction, disjunction, the quantifiers, and identity. Thus restricting classical logic will tend to impose widespread restrictions on its explanatory power, by blocking the derivation of its classical consequences in particular applications.'
}

Australasian Journal of Logic (16:7) 2019, Article no. 4 
counterparts. Consider again the paracomplete theories. The negation of Strong Kleene is a three-valued operator, but it generalizes the classical negation in the sense that it remains classical for boolean values. More generally, a number of nonclassical concepts can be characterized by the following generalization of classical concepts:

$$
\begin{aligned}
& f_{\neg}(x)=1-x ; \\
& f_{\wedge}(x, y)=\operatorname{Min}(x, y) ; \\
& f_{\vee}(x, y)=\operatorname{Max}(x, y) .
\end{aligned}
$$

The generalized logical concepts are versatile. They allow formalization of theories that would be inconsistent with classical concepts, but they all the same behave classically in the limit case where all sentences take boolean values.

But the nonclassical response does not depend on nonclassical concepts being generalizations of classical concepts. It is also possible to adopt a logical theory that includes classical concepts, say, truth-functional connectives. However, a nonclassical theory need not at the same time provide for these connectives to underpin valid arguments in general. They might for example be limited to certain language fragments or certain theoretical applications.

There is a worry that in some systems two connectives cannot cohabitate without collapse. The standard example is that intuitionistic negation becomes equivalent to classical negation in a natural deduction setting. But such nonconservativeness results are artifacts of formal systems, varying from proof system to proof system. In any case, there is no problem with a logical theory, in the sense prescribed in Section 2, containing both expressions, given that their interaction is suitably controled. We are, after all, perfectly capable of theorizing about and comparing the two negations.

None of this is likely to convince anyone to drop their allegiance to the classical theory. But that is not the current aim. It is simply to illustrate that there are ways of combining classical mathematics with nonclassical logics. The classical theory might ultimately be preferable because of considerations such as simplicity, but not because the theory is indispensable to mathematics. We should, therefore, reject Premise 2 in the argument above, and with it the conclusion that classical logic is indispensable to our best theories. Although it is not ruled out that there are other indispensability arguments available to the classical logician, it strikes me as unlikely. In any case, if the classical theory has a priviliged role to play in non-logical sciences other than mathematics, the case must be made. In the meantime, I conclude 
that whatever evidential confirmation there is for the classical theory, it is not forthcoming from an indispensability argument. I am not, however, identifying a particular nonclassical theory that is indispensable to our best theories. Indispensability is a legitimate source of evidential confirmation for the anti-exceptionalist, but one that presently does not help us select a logical theory.

\section{Non-evidential criteria}

Even if the preceding discussion does not exhaust all possible sources of evidence, I think it includes at least some of the types of evidential confirmation compatible with anti-exceptionalism. The problem is that none of the potential sources offer evidence that unambiguously points to a unique logical theory. More specifically, therefore, we cannot conclude that the classical theory is a better fit with the evidence than its rival nonclassical theories. True, no nonclassical alternative stands out either, but the above discussion should nonetheless temper the claim that anti-exceptionalism strongly supports the classical theory.

Still, abductive arguments do not depend on fit with the evidence alone. Abduction allows for a number of weighted criteria that together form the basis for theory choice. So even if the choice of logical theory cannot be made on the basis of fit with the evidence, the classical theory might score better on non-evidential criteria. What could these criteria be? There is a well-known laundry list of virtues from philosophy of science, for example, simplicity, strength, elegance, unifying power, and conservativeness. These are in fact the criteria that Priest $(2014,217)$ and Williamson $(2017,14)$ suggest as reasonable companions to the criterion that the theory should fit the evidence.

Let us start with some remarks on conservativeness. As a criterion of theory-choice, conservativeness is in a sense upstream from the evidential sources. On the assumption that the classical theory is our current logical theory, conservativeness says that it has a pre-evidential advantage. We can think of this as a preference ordering where the classical theory is ranked first at the initial stage, and where abductive arguments reorder the preferences at later stages. Unless there is sufficient evidence to dislodge the classical theory from its prior position, no revision is called for. The burden of proof, the conservative concludes, is on the nonclassicist. We have seen how difficult it is to to support an outright rejection of a validity claim, so the advantage

Australasian Journal of Logic (16:7) 2019, Article no. 4 
is decisive. ${ }^{28}$

If one accepts conservativism, and also that the classical theory is indeed our current theory, then there is a reason to retain the classical theory if the evidence is inconclusive. I don't want to argue against that methodology here. What is important is that conservativeness is not normally part the grounds offered for the classical theory. When Quine, Williamson and other classically-minded anti-exceptionalists defend the classical theory, they rest their case on other criteria. Williamson, for example, rejects the conservative approach, instead relying on intrinsic features of classical logic: ${ }^{29}$

The strong prima facie abductive case for classical logic just noted does not depend on a principle of conservativism. It does not rely on the position of classical logic as the status quo, the logic we more or less currently accept, nor does it appeal to the benefits of familiarity or the costs of change. It concerns intrinsic features of classical logic, such as simplicity and strength, which it would have even if we currently accepted some non-classical logic. (Williamson 2017, 20)

Quine extols the virtues of classical logic in similar terms:

Classical quantification theory enjoys an extraordinary combination of depth and simplicity, beauty and utility. It is bright within and bold in its boundaries. Deviations from it are likely, in contrast, to look rather arbitrary. (Quine 1969, 112-13)

The question, then, is whether Quine and Williamson are right in thinking that the non-evidential criteria unequivocally support the classical theory. I don't think that is the case, in fact, far from it. Let us look at two of the most frequently cited theoretical virtues: strength and simplicity.

Previously I have argued that strength - whatever it means in the context of logical theories - does not straightforwardly count in the favour of the classical theory (Hjortland 2017). I don't want to recount the arguments in detail here, but the gist is that the strength of a logical theory should not

\footnotetext{
${ }^{28}$ In a related discussion, Harman $(1984,114)$ formulates a principle of conservativism as a criterion in inference to the best explanation: 'Conservatism is a factor in the sense that one should not change one's view without a positive reason for doing so and, in changing one's view, other things being equal, one ought to minimize such change.'

${ }^{29}$ See also Williamson $(1994,186)$ : '[C]lassical semantics and logic are vastly superior to the alternatives in simplicity, power, past success, and integration with theories in other domains.'
}

Australasian Journal of Logic (16:7) 2019, Article no. 4 
be equated with the deductive strength of a consequence relation. First, the consequence relation is only one component of a logical theory. A logical theory features claims about a number of properties, such as consistency, provability, negations, conditionals, and many others. Second, deductive strength is not a measure of explanatory power, the property of strength that we most frequently associate with theory selection. And, third, subclassical theories are deductively weaker for a reason, in fact it is their very raison d'être. Only by restricting deductive strength can the subclassical theories be consistently combined with theories of unrestricted truth or naïve comprehension. Finally, a reduction in deductive strength typically leads to an increase in expressive power. In short, there is no shortcut to claiming that the classical theory is stronger than its nonclassical rivals. It is certainly not something nonclassical logicians should concede without further ado.

What about simplicity? Both Williamson and Quine mention simplicity as one of the chief virtues of classical logic. Is there a case to be made for simplicity being a tie-breaker in favour of classical logic? The immediate problem is that it is not at all clear what the simplicity measure is supposed to be. First of all, while simplicity ought to be a measure of the logical theory in its entirety, it is typically used to refer to some formal property of a logical system (e.g. classical model theory or a classical proof system).

Second, even if we let the misapplication slide, it is still not clear what the measure is supposed to be. A first stab is that we are talking about the simplicity of proofs. In that case, the claim might be that classical proofs are simpler than nonclassical proofs. That is a line of thought with some precedent. In the context of formal theories of truth, Feferman (1984, 95), for example, states that 'nothing like sustained ordinary reasoning' can be carried out in non-classical logics. If ordinary reasoning is classical, then Feferman is right. However, many nonclassical logicians deny the antecedent. Their point is precisely that classical logic cannot account for ordinary reasoning with, say, vague expressions. More likely, Feferman has in mind something like mathematical proofs. I agree that there are mathematical proofs that require instances of classical laws. What is unclear, however, is whether this means that nonclassical theories cannot accommodate the classical proofs as special cases (see Section 6).

The simplicity of proofs is a problematic measure for other reasons. Whether a derivation is simpler than another tends to be a highly systemdependent matter. For example, nonclassical logics often have fewer proof rules than classical systems. Surely that is one sense in which the nonclassical systems are simpler. Of course, fewer proof rules might mean that finding a proof is harder, or even impossible, but why should that count against 
the nonclassical system? After all, the nonclassical theory typically discards the classical proof rules for a reason (e.g. they lead to inconsistency in the presence of certain non-logical principles). Put more rhetorically, a simple proof for a falsehood is too much simplicity.

The simplicity could also be a measure of the models or some modeltheoretic property. Classical models are simple: there are only two truth values, one designated value, and the logical connectives are interpreted by truth-functions. In contrast, nonclassical models have multiple truthvalues (sometimes continuum-many), several designated values, intensional interpretations of connectives, and other unlovely complications. There is a reason why nonclassical logics are not part of a first course in logic. Typically they extend or generalize classical formalism in various ways. This does not mean, however, that the nonclassical model theory is unnecessarily complicated. Just like classical derivations have more components than their nonclassical counterparts, nonclassical models have more components than their classical counterparts. Classical derivations allow for more proofs, and, correspondingly, nonclassical models allow for more counter-examples, which in turn allow for greater expressive power.

Simplicity just isn't a univocal criterion. On the contrary, it is a multidimensional measure, if anything, and one that does little to support the classical theory. That shouldn't be a surprise. Even if simplicity is a theoretical virtue, it is not one that we should assign a lot of weight. Rather, the lesson from scientific theories is that simpler theories are often rejected in favour of complications that affords explanatory strength. Classical mechanics is simple, and since it is still applicable in many cases (e.g. macroscopic objects at low speed), it merits its inclusion in textbooks. That does not change the fact that it is a theory with shortcomings that can only be addressed by introducing complications, for instance, the complications of quantum mechanics. The nonclassical logicians offer a similar picture. The classical theory has elegant applications to logical properties in special cases (e.g. mathematics), but it is a theory whose shortcomings can only be addressed by the introduction of complications, specifically, the complications of nonclassical theories. Even if the nonclassical theories ultimately are not selected, simplicity should not be the reason.

\section{Conclusion}

I have developed and discussed three accounts of evidential confirmation of logical theories, compatible with anti-exceptionalism. I argue that the 
available evidence undercuts the received view, championed by Quine, that anti-exceptionalism provides a justification for the classical theory. It doesn't. Anti-exceptionalism restricts the type of argument that can be given for a logical theory, with the result that the choice of classical logic looks less obvious. Of course, no one can deny that the classical theory is unusually dominant, to the extent that it is rarely considered a mere theory. But when we turn the anti-exceptionalist's scrutiny to logic, we do not find sufficient grounds for its position of strength. Classical logic is an elegant and diverse formal tool, but that alone cannot undergird the classical theory as a theory of logical properties.

\section{References}

Arnold, J. and Shapiro, S. (2007). Where in the (world wide) web of belief is the law of non-contradiction? Noûs, 41(2):276-297.

Birkhoff, G. and Von Neumann, J. (1937). The logic of quantum mechanics. Journal of Symbolic Logic, 2(1):44-45.

Boghossian, P. (2000). Knowledge of logic. In Boghossian, P. and Peacocke, C., editors, New Essays on the A Priori, pages 229-254. OUP, Oxford.

Bueno, O. and Colyvan, M. (2004). Logical non-apriorism and the law of non-contradiction. In Priest, G., Beall, J., and Armour-Garb, B., editors, The law of non-contradiction: New philosophical essays, pages 156-175. OUP, Oxford.

Carnap, R. (1937). The logical syntax of language. Routledge \& Kegan Paul Ltd, New York.

Colyvan, M. (2001). The Indispensability of Mathematics. Oxford University Press, Oxford.

Dummett, M. (1991). The logical basis of metaphysics. Harvard University Press, Cambridge, MA.

Enoch, D. and Schechter, J. (2008). How are basic belief-forming methods justified? Philosophy and Phenomenological Research, 76(3):547-579.

Evans, J. S. B., Barston, J. L., and Pollard, P. (1983). On the conflict between logic and belief in syllogistic reasoning. Memory $\&$ cognition, 11(3):295-306.

Feferman, S. (1984). Toward useful type-free theories. I. Journal of Symbolic Logic, 49:75-111.

Field, H. (1989). Realism, mathematics, and modalty. Blackwell, Oxford.

Field, H. (2000). Apriority as an evaluative notion. In Paul Boghossian, 
C. P., editor, New Essays on the A Priori, pages 117-149. Clarendon Press, Oxford.

Field, H. (2008). Saving Truth from Paradox. OUP, Oxford.

Field, H. (2009). What is the normative role of logic? Proceedings of the Aristotelian Society Supplementary Volume, LXXXIII:251-268.

Field, H. (2015). What is logical validity? In Caret, C. and Hjortland, O. T., editors, Foundations of Logical Consequence, pages 33-70. OUP, Oxford.

Glanzberg, M. (2015). Logical consequence and natural language. In Caret, C. and Hjortland, O., editors, Foundations of Logical Consequence, pages 71-120. OUP, Oxford.

Griggs, R. and Cox, J. (1982). The Elusive Thematic-Materials Effect in Wason. British Journal of Psychology, 73(3):407-20.

Halbach, V. and Horsten, L. (2006). Axiomatizing Kripke's theory of truth. Journal of Symbolic Logic, 71(2):677-712.

Harman, G. (1984). Logic and reasoning. Synthese, 60(1):107-127.

Harman, G. (1986). Change in view: Principles of reasoning. MIT Press, Cambridge, MA.

Hjortland, O. T. (2014). Speech acts, categoricity, and the meanings of logical connectives. Notre Dame Journal of Formal Logic, 55(4):445-467.

Hjortland, O. T. (2017). Anti-exceptionalism about logic. Philosophical Studies, 174(3):631-658.

Hjortland, O. T. (2019). Disagreement about logic. Inquiry. Forthcoming.

Horsten, L. (2011). The Tarskian Turn: Deflationism and Axiomatic Truth. MIT Press, Cambridge, MA.

Kripke, S. (1975). Outline of a theory of truth. Journal of Philosophy, $72(19): 690-716$.

MacFarlane, J. (2004). In what sense (if any) is logic normative for thought. Delivered at the American Philosophical Association Central Division meeting.

Maddy, P. (2002). A naturalistic look at logic. Proceedings and addresses of the American Philosophical Association, 76(2):61-90.

Maddy, P. (2014). The Logical Must: Wittgenstein on Logic. Oxford University Press.

Maudlin, T. (2005). The tale of quantum logic. In Ben-Menahem, Y., editor, Hilary Putnam, chapter 6, pages 156-187. CUP, Cambridge.

McGee, V. (1985). A counterexample to modus ponens. Journal of Philosophy, 82(9):462-471.

Morley, N. J., Evans, J. S. B., and Handley, S. J. (2004). Belief bias and figural bias in syllogistic reasoning. Quarterly Journal of Experimental Psychology Section A, 57(4):666-692. 
Priest, G. (1987/2006b). In Contradiction. OUP, Oxford, 2nd edition.

Priest, G. (2006a). Doubt Truth to be a Liar. OUP, Oxford.

Priest, G. (2008). An Introduction to Non-Classical Logic. Cambridge University Press, Cambridge, 2nd edition.

Priest, G. (2014). Revising logic. In Rush, P., editor, The Metaphysics of Logic, chapter 12, pages 211-223. CUP, Cambridge.

Priest, G. (2016). Logical disputes and the a priori. Logique et Analyse, $59(236): 347-66$.

Putnam, H. (1969). Is logic empirical? In Cohen, R. S. and Wartofsky, M. W., editors, Boston Studies in the Philosophy of Science: Proceedings of the Boston Colloquium for the Philosophy of Science 1966/1968, pages 216-241. Springer, Dordrecht.

Putnam, H. (1971). Philosophy of logic. Harper \& Row, New York.

Putnam, H. (1981). Quantum mechanics and the observer. Erkenntnis, 16(2):193-219.

Putnam, H. (2012). The curious story of quantum logic. In Caro, M. D. and Macarthur, D., editors, Philosophy in the Age of Science: Physics, Mathematics, and Skepticism, pages 162-177. Harvard University Press, Cambridge, MA.

Quine, W. V. (1951). Two dogmas of empiricism. Philosophical Review, $60(1): 20-43$.

Quine, W. V. (1969). Existence and quantification. In Ontological relativity and other essays, pages 91-113. Columbia University Press, New York.

Restall, G. (1994). On logics without contraction. PhD thesis, Department of Philosophy, University of Queensland.

Restall, G. (2005). Multiple conclusions. In Hájek, P., Valdés-Villanueva, L., and Westerståhl, D., editors, Logic, Methodology and Philosophy of Science, pages 189-205. College Publications.

Russell, B. (1918). The philosophy of logical atomism. Monist, 28:495-527.

Russell, G. (2008). One true logic? Journal of Philosophical Logic, 37(6):593611.

Russell, G. (2015). The justification of the basic laws of logic. Journal of Philosophical Logic, 44(6):793-803.

Russell, G. K. (2014). Metaphysical analyticity and the epistemology of logic. Philosophical Studies, 171(1):161-175.

Scharp, K. (2013). Replacing truth. Oxford University Press, Oxford.

Schechter, J. and Enoch, D. (2006). Meaning and justification: The case of modus ponens. Noûs, 40(4):687-715.

Shapiro, S. (2014). Varieties of logic. Oxford University Press, USA.

Sider, T. (2013). Writing the Book of the World. OUP, Oxford. 
Steinberger, F. (2016). Explosion and the normativity of logic. Mind, 125(498):385-419.

Steinberger, F. (2019). Three ways in which logic might be normative. The Journal of Philosophy, 116(1):5-31.

Wason, P. (1966). Reasoning. New horizons in psychology, 1:135-151.

Wason, P. and Shapiro, D. (1971). Natural and contrived experience in a reasoning problem. The Quarterly Journal of Experimental Psychology, 23(1):63-71.

Weber, Z. (2010). Transfinite numbers in paraconsistent set theory. The Review of Symbolic Logic, 3(01):71-92.

Weber, Z. (2012). Transfinite cardinals in paraconsistent set theory. The Review of Symbolic Logic, 5(02):269-293.

Williamson, T. (1994). Vagueness. Routledge, London.

Williamson, T. (2007). The Philosophy of Philosophy. Blackwell, Oxford.

Williamson, T. (2013a). Logic, metalogic and neutrality. Erkenntnis, $79(2): 211-231$.

Williamson, T. (2013b). Modal logic as metaphysics. Oxford University Press, Oxford.

Williamson, T. (2017). Semantic paradoxes and abductive methdology. In Armour-Garb, B., editor, The Relevance of the Liar. OUP, Oxford. Forthcoming.

Woods, J. (2019). Logical partisanhood. Philosophical Studies, 176(5):12031224.

Wright, C. (1986). Inventing logical necessity. In Butterfield, J., editor, Language, Mind, and Logic, pages 187-209. Cambridge University Press, Cambridge.

Zardini, E. (2014). Context and consequence. an intercontextual substructural logic. Synthese, 191(15):3473-3500. 